\title{
Caregiver strain in spouses of stroke patients
}

Holly Blake, Nadina B Lincoln and David D Clarke

School of Psychology, University of Nottingham, Nottingham, UK.

Address for correspondence:

Professor Nadina B Lincoln,

School of Psychology, University of Nottingham, Nottingham

NG7 2RD, UK.

e-mail: nbl@psychology.nottingham.ac.uk

Received 21st October 2001; returned for revisions 27th November 2001; manuscript accepted 27th December 2001. 


\section{Abstract}

Objective: To test the ability of a previously generated logistic regression model to predict caregiver strain from carer mood, negative affectivity and perceived patient functional ability.

Design: Postal prospective survey.

Setting: Spouses of community-residing patients identified from hospital stroke registers.

Method: Spouses were assessed at three and six months after stroke. A previously derived equation was used to make predictions at three months of their level of strain at six months, which were compared with observed outcomes.

Measures: Spouses were asked to complete the Caregiver Strain Index (CSI), the General Health Questionnaire (GHQ-12), the Positive and Negative Affectivity Schedule (PANAS) and to assess patients' independence in activities of daily living on the Extended Activities of Daily Living Scale (EADL).

Results: Of 409 stroke patients, 276 had an identifiable co-resident spouse and 116 (42\%) completed the measures. At three months after stroke, 39 carers (34\%) were under significant strain with $40(35 \%)$ under strain at six months. The predictive model using the GHQ-12, PANAS and EADL at three months was $78 \%$ accurate in predicting levels of caregiver strain at six months.

Conclusion: Carers at risk of later strain could be identified for further follow-up. Services to provide emotional support to carers might be effective in the reduction of carer strain. 


\section{Introduction}

Carers of stroke patients experience significant strain. [1,2] The early identification of carers who may be unable to cope later after stroke would enable effective targeting of support to prevent carer strain. In a previous study [1] of factors associated with strain, we assessed caregiver perceptions of strain, stress, mood, handicap, adjustment, social support, life satisfaction and personality, and patient's independence in activities of daily living.[1] The results of this work suggested that carer characteristics were important in the experience of strain. Strain was most closely related to caregiver mood, caregiver's perceptions of their partner's abilities in extended activities of daily living and negative affectivity.[1]

Mood refers to a transient, 'here and now' emotional state, which may be amenable to therapeutic intervention. Negative affectivity (NA) is a general factor of subjective distress. It represents the extent to which a person experiences negative mood states, including upset, angry, worried, guilty, afraid and disgusted. High NA individuals often report distress, discomfort and dissatisfaction over time regardless of the situation, even in the absence of any overt or objective source of stress. Research has suggested that NA is related to self-reported stress and less adaptive coping strategies[3] and is a vulnerability factor for the development of anxiety and depression. [4] High NA has been associated with caregiver strain in other patient groups.[5] Other research has also demonstrated that poor patient independence is associated with negative carer outcomes. For example, one study showed that disability on the Barthel Index was related to increased carer overload.[6] However, predictive interpretations of our previous data[1] were not possible as the measures were taken concurrently. The previous study was a cross-sectional design and in order to confirm the results, the findings needed to be tested prospectively, on a 
new sample of carers. The primary aim of the study was to ascertain the accuracy of our logistic regression model in predicting carers under strain. This would enable early identification of those carers who were most likely to suffer from strain later on. Furthermore, consistent findings in more than one study showing an association between a risk factor and an outcome make it more likely that the association is not the artefact of one study.[7]

\section{Methods}

Ethical approval was granted for postal contact of carers, and telephone contact or home visits to carers when it was requested. Co-resident spouses of patients on stroke registers at three centres were identified using the Patient Administration System (PAS), which contained information about marital status and demographic information of the patient. Further information about the carer was not available from the registers. The name and telephone number of each patient's general practitioner (GP) was extracted from this register, and all GPs were contacted in order to check whether the patient was still alive and whether the address and marital status had changed since the recording of the register.

As the study was designed to prospectively test the model developed in previous research,[1] factors previously identified as important were assessed using a largeprint questionnaire format. These were posted to the spouses three months after stroke, together with a covering letter explaining the voluntary nature of the research and a reply-paid envelope. Information was collected by postal questionnaire unless the carer required help with completion of the forms. In these situations home visits were made or help was provided over the telephone. Help was given reading the questions or writing the answers, but no extra information was provided, so that data 
from these questionnaires was comparable with that received through the post. The assessment package included the Caregiver Strain Index (CSI) [2] as a measure of caregiver strain, the General Health Questionnaire-12 (GHQ-12) [8] as a measure of mood, the Extended Activities of Daily Living Scale (EADL) [9] as a measure of patient functional abilities and the Positive and Negative Affectivity Schedule (PANAS) [10] as a measure of dispositional positive and negative tendencies. The CSI, GHQ-12 and PANAS were completed by the caregiver with reference to themselves. The carers were asked to complete the EADL in terms of how they perceived their partner's abilities. Spouses were sent the CSI to complete again six months after the stroke.

Predicted outcomes were calculated using the regression equation previously developed and compared with actual levels of strain at six months (observed outcome) to provide an indication of the accuracy and error levels of the regression model developed previously. A discriminant analysis was adopted to distinguish those carers who were likely to suffer from strain. First, L was defined as the logit of the probability $\mathrm{p}$ that a carer would be under significant strain. The values for $\mathrm{L}$ were calculated for each carer using the regression coefficients from the original model:

$\mathrm{L}=-2.05+(0.30 *$ GHQ-12 $)-(0.13 *$ EADL $)+(0.08 *$ PANASNA $)$

A new dichotomous variable was created to represent the model predictions. If the probability of being under strain was greater than the probability of not being under strain, carers were predicted as being strained, i.e., 'under strain' was equated with 'p $>1 / 2^{\prime}$. The number of carers for whom the observed outcome was correctly predicted by the regression model was calculated and divided by the total number to provide an 
indicator of accuracy.

\section{Results}

A total of 409 patients were identified from the registers as being alive, married and living in the community. Of the 409 patients and their spouses, $133(33 \%)$ couples were excluded. This was because one patient had moved away, seven had poor English, three were separated or divorced, 39 patients died, 14 were in institutional care, 37 spouses died, six spouses were too ill, four spouses were in institutional care and 22 were omitted when the study was temporarily suspended at Nottingham City Hospital (NCH).

Altogether, $276(67 \%)$ were considered for inclusion in the study. The three-month form was returned by 130 (47\%) spouses and the six-month form was returned by 116 (42\%) spouses. There were $116(42 \%)$ spouses who returned both the three- and sixmonth questionnaires. Of these carers, 33 (28\%) were men and $83(72 \%)$ were women. Carer age ranged from 38 to 85 years (mean 66.35, SD 10.8). Of the patients, $83(72 \%)$ were men and $33(28 \%)$ were women. Patient age ranged from 45 to 88 years (mean 68.63, SD 10.53). The median CSI score at both three and six months

was 5. Using the Wilcoxon Signed Ranks Test, no significant differences were found between strain in the spouse at three and six months $(\mathrm{z}=-0.56, \mathrm{p}=0.57)$. Three- and six-month strain were significantly correlated $(\mathrm{rs}=0.86, \mathrm{p}<0.01)$.

Distribution of strain was examined separately at three and six months. Spouses who scored equal to or greater than 7 on the CSI were considered to be under significant strain.[2] At three months, 45 spouses were strained (39\%) and 71 were not strained (61\%). At six months, 46 spouses were strained (40\%) and 70 were not strained $(60 \%)$. There was no significant difference between men and women spouses in the 
level of strain at either three months $(U=1233, p=0.40)$ or six months $(U=1293.5$, $\mathrm{p}=0.64$ ). Frequencies (and percentages out of total completed) of this group's responses of 'yes' to each of the items on the CSI at both three and six months are shown in Table 1.

At three months, changes in personal plans were reported by the greatest percentage of spouses, followed by feeling overwhelmed and feeling that the patient had changed from his/her former self. At six months, the items selected by the greatest percentage of spouses were feeling overwhelmed and changes in personal plans, followed by upsetting behaviours and feelings of confinement. For the majority of items, the percentage of spouses selecting the item remained relatively constant from three months to six months. However, there was a notable drop in the percentage of carers reporting family changes by six months, and a notable increase in the percentage of carers reporting financial strain. The least frequently reported were work adjustments and physical strain.

The distribution of scores on each questionnaire measure is shown in Table 2. As the majority of data were ordinal, nonparametric Spearman rank correlation coefficients were calculated. Each variable measured at three months was correlated against strain on the CSI at six months. Strain at six months was significantly correlated with GHQ$12(\mathrm{rs}=0.72, \mathrm{p}<0.001)$, patient EADL $(\mathrm{rs}=-0.65, \mathrm{p}<0.001)$ and negative affectivity $(\mathrm{rs}=0.66, \mathrm{p}<0.001)$. Predicted outcomes and three-month CSI were tabulated against observed outcomes and the results are shown in Table 3. Ninety (78\%) carers were accurately predicted as being either strained or not strained on the CSI at six months using the previously generated model. Allowing for agreement due to chance the correspondence was poor (kappa $=0.53, \mathrm{p}<$ 
0.001). The equation was equivalent at predicting those under strain $(33 / 46=72 \%)$ and those not under strain $(57 / 69=83 \%)$. As three and six month CSI were significantly correlated the same analysis was conducted using the CSI at three months to predict carer strain at six months.

Eighty-five per cent of spouses who were accurately predicted by three-month CSI as being strained or not strained on the CSI at six months and correspondence was good $($ kappa $=0.70, \mathrm{p}<0.001)$.

\section{Discussion}

In this sample, levels of strain were high with $39 \%$ under strain at three months and $40 \%$ under strain at six months. This supports the view that strain remains relatively high over time [11] and is consistent with prevalence rates found in other studies of stroke caregivers.[12]

These results supported our previous findings [1] and demonstrated that mood, negative affectivity and perceived EADL were useful in predicting strain in carers six months after stroke. Therefore, important factors appear to be the spouse's appraisal of their partner's disability, together with two emotional components of caregiver subjective well-being, one transient and one stable.

These findings support other work on carer strain that has demonstrated a relationship between emotional distress and caregiver strain, both in stroke [13-15] and other patient groups.[5] Spouses who cared for patients whom they perceived as dependent in extended activities of daily living were more likely to experience strain. It is well documented that patient functional ability exerts some influence on strain. [6][16] However, there may be differences between objective measures of patient disability 
and inferences made by the caregiver. The problems of using proxy ratings of the patients' functional abilities have been recognized for some time [17][18]. It may be that patients who are more dependent in EADL have spouses who are more strained. Alternatively, it may be that spouse's perceptions of their partner's abilities are distorted, perhaps from either a misunderstanding of stroke or from unrealistic expectations about recovery, and that these distorted beliefs are the factor that is related to strain. Clarification of this issue would require an assessment of the discrepancy between carer's ratings of the patient's function and objective measures of functional ability.

The relationship between negative affectivity and caregiving strain supports work done in other patient groups.[5] As a general factor of subjective distress it is logical that high negative affectivity would be associated with high levels of strain.

High NA individuals may be more introspective and dwell on their shortcomings, thus rendering them prone to depression. In addition, high NA individuals have a less favourable view of themselves and others and so may be more likely to appraise their partner as being less independent than they actually are.

Identifying carers who demonstrate these three factors may enable the recognition of those carers who are likely to suffer from high levels of strain. This would allow the identification of those carers towards whom intervention should be targeted. Although the model was relatively accurate in predicting carers at risk from strain, the $23 \%$ margin of error suggests that other factors may also be involved. The margin of error might be explained by other factors not addressed, such as incontinence, communication problems and disrupted sleep.

Given the significant correlation between carer strain at three and six months and the greater accuracy of predicting carer strain at six months from carer strain at three 
months it may seem simpler to identify carers who may be strained later on by assessing strain early after stroke. However, at three months, many patients are still in hospital and therefore items of the CSI may not be relevant during this stage, for example, experiencing financial strain or physical strain. For these carers it may be more relevant to assess mood, negative affectivity and perceived independence of the patient to predict later strain. Although the results suggest that a spouse's appraisal of their partner's disability together with a knowledge of transient and stable emotional well-being early after stroke would enable us to predict levels of strain which may be experienced later on, this association is not necessarily causal. Furthermore, early CSI scores predicted later CSI scores with greater accuracy than our model. From a pragmatic point of view, it may be simpler to measure strain early after stroke to detect those spouses who may be under strain later on. However, our equation is useful in that it provides more information as to which factors or characteristics are associated with high levels of strain. Knowledge of strain alone would not provide any indication as to which forms of intervention might be most successful at reducing strain.

There are a number of methodological limitations. As the spouses were predominantly elderly it is possible that some carers could not complete the forms due to their own cognitive impairments, or were themselves too ill to take part in the study. Therefore the sample may be biased in the direction of a healthy population. However, this is unlikely as patient names were taken from a hospital register, which may have resulted in a reduced number of carers whose spouses had only a very mild stroke with little or no resulting de.cits. Furthermore, the response rate is comparable 
with our previous work [1] and with similar research on stroke carers using similar methodology. [19] In addition, a low response rate is not unexpected in a group that has high mortality and a high rate of movement into residential care.

Home visits could have increased the response rate rather than postal collection of data, but this is more expensive and time-consuming. There was no control group and therefore it is impossible to tell whether strain levels in this sample were higher than they would have been in a non-caregiving group. Three and six months were selected as appropriate because three months is still during the acute stage, although many of the patients have returned home by this time and six months is long enough for carers to be aware of all the problems that occur. Assessment at one year would have been useful as by then, optimal levels of functional capacity would be expected in the patient. However, this may have reduced case numbers due to death and disability.

\section{Clinical messages}

- Strain is predicted by carer mood (both state and trait) and carer's perceptions of patient independence in EADL.

- Identifying and monitoring 'at risk' carers may prevent situations reaching crisis stage.

- Intervention to improve mood and provide emotional support may be effective in reducing strain.

\section{Acknowledgements}

This research was funded by REMEDI. The authors would like to thank all the carers who assisted with this research and Dr John Gladman (Queen's Medical Centre), 
Professor Philip Bath (Nottingham City Hospital) and Dr Tom Robinson (Leicester General Hospital) for access to the stroke registers at the participating hospitals.

\section{References}

1 Blake H, Lincoln NB. Factors associated with strain in co-resident spouses of patients following stroke. Clin Rehabil 2000; 14: 307-14.

2 Robinson B. Validation of a Caregiver Strain Index. J Gerontol 1983; 38: 344-48.

3 Gunthert K, Cohen L, Armeli S. The role of neuroticism in daily stress and coping. J Pers Soc Psychol 1999; 77: 1087-100.

4 Watson D, Clarke L. The PANAS-X: Manual for the Positive and Negative Affectivity Schedule - expanded form. City of Iowa: University of Iowa, 1994.

5 Blake H. Strain and psychological distress among informal supporters of reflex sympathetic dystrophy patients. Disabil Rehabil 2000; 22: 827-32.

6 Carod-Artal F, Egido-Navarro J, Gonzalez-Gutierrez J, Seijas EVd. Perception of long-term overload in caregivers of patients who have survived a stroke. Rev Neurol 1999; 28: 1130-38.

7 Garb J. Understanding medical research: a practitioner's guide. New York: Little, Brown and Co., Inc., 1996. 
8 Goldberg D, Williams P. A user's guide to the General Health Questionnaire.

Windsor: NFER Nelson, 1988.

9 Nouri F, Lincoln N. An extended activities of daily living scale for stroke patients.

Clin Rehabil 1987; 1: 301-305.

10 Watson D, Clark L, Tellegen A. Development and validation of brief measures of positive and negative affectivity: The PANAS scales. J Pers Soc Psychol 1988; 54: $1063-70$.

11 Langton Hewer R. Rehabilitation after stroke. Q J Med 1990; 76: 659-74.

12 Dennis M, O'Rourke S, Lewis S, Sharpe M, Warlow C. A quantitative study of the emotional outcome of people caring for stroke survivors. Stroke 1998; 29: 1867-72.

13 Mitchley N, Gray J, Pentland B. Burden and coping among the relatives and carers of brain injured survivors. Clin Rehabil 1996; 10: 3-8.

14 Op Reimer S, Haan Rd, Rijnders P, Limburg M, Bos Gvd. The burden of caregiving in partners of long-term stroke survivors. Stroke 1998; 29: 1605-11.

15 Draper B, Poulos C, Cole A, Poulos R, Ehrlich F. A comparison of caregivers for elderly stroke and dementia victims. J Am Geriatr Soc 1992; 40: 896-901. 
16 Santariano W, Minkler M, Langhauser C. The significance of an ill-spouse for assessing health differences in an elderly population. J Am Geriatr Soc 1984; 35: 405.

17 Andrews K, Stewart J. Stroke recovery: he can, but does he? Rheumatol Rehabil 1979; 18: 43-48.

18 Spranger M, Aaronson N. The role of health care providers and significant others in evaluating the quality of life of patients with chronic disease: a review. J Clin Epidemiol 1992; 45: 743-60.

19 Pound P, Gompertz P, Ebrahim S. Development and results of a questionnaire to measure carer satisfaction after stroke. J Epidemiol Commun Health 1993; 47: 500505. 
Table 1 Responses to individual items on the Caregiver Strain Index

\begin{tabular}{lll}
\hline Item on CSI & \multicolumn{2}{l}{$\begin{array}{l}\text { Number responding 'Yes' } \\
(n=116)\end{array}$} \\
\cline { 2 - 3 } & Three months & Six months \\
\hline Disturbed sleep & $47(41 \%)$ & $48(41 \%)$ \\
Inconvenient & $38(33 \%)$ & $32(28 \%)$ \\
Physical strain & $25(22 \%)$ & $29(25 \%)$ \\
Confining & $59(51 \%)$ & $58(50 \%)$ \\
Family changes & $49(42 \%)$ & $41(35 \%)$ \\
Changes in personal plans & $63(54 \%)$ & $59(51 \%)$ \\
Other demands & $35(30 \%)$ & $39(34 \%)$ \\
Emotional adjustments & $36(31 \%)$ & $41(35 \%)$ \\
Upsetting behaviour & $58(50 \%)$ & $58(50 \%)$ \\
Patient has changed & $60(52 \%)$ & $57(49 \%)$ \\
Work adjustment & $23(20 \%)$ & $20(17 \%)$ \\
Financial strain & $44(38 \%)$ & $47(41 \%)$ \\
Feeling overwhelmed & $62(53 \%)$ & $59(51 \%)$ \\
\hline
\end{tabular}


Table 2 Distribution of scores on carer assessments

\begin{tabular}{|c|c|c|c|c|c|c|}
\hline \multirow[t]{3}{*}{ Variable } & \multirow{3}{*}{$\begin{array}{l}\text { Possible } \\
\text { range }\end{array}$} & \multirow[t]{3}{*}{$n$} & \multicolumn{4}{|c|}{ Time after stroke } \\
\hline & & & \multicolumn{2}{|c|}{3 months } & \multicolumn{2}{|c|}{6 months } \\
\hline & & & Median & IQR & Median & IQR \\
\hline $\mathrm{CSI}$ & $0-13$ & 116 & 5 & $1-8$ & 5 & $1-8$ \\
\hline Patient EADL & $0-22$ & 116 & 11 & $3-19$ & 13 & $5-19$ \\
\hline Carer GHQ-12 & $0-12$ & 116 & 3 & $0-7$ & 1 & $0-6$ \\
\hline Negative affectivity & $10-50$ & 116 & 19 & $13-29$ & 17 & $13-29$ \\
\hline Positive affectivity & $10-50$ & 116 & 33 & $26-37$ & 33 & $26-37$ \\
\hline
\end{tabular}

IQR, interquartile range; CSI, Caregiver Strain Index; EADL, Extended Activities of Daily Living Scale; GHQ-12, General Health Questionnaire-12.

Table 3 Cross-tabulation of predicted outcome with observed outcome and three-month CSI with observed outcome

\begin{tabular}{lccc}
\hline & \multicolumn{2}{c}{ Observed outcome (six-month CSI) } \\
\cline { 2 - 4 } & Not strained & Strained & Total \\
\hline $\begin{array}{l}\text { Predicted outcome from model (predicted CSI) } \\
\text { Not strained }\end{array}$ & $57(49 \%)$ & $13(11 \%)$ & 70 \\
Strained & $13(11 \%)$ & $33(29 \%)$ & 46 \\
Total & 69 & 46 & 116 \\
Three-month CS & $61(53 \%)$ & $9(7 \%)$ & 70 \\
Not strained & $8(7 \%)$ & $38(33 \%)$ & 46 \\
Strained & 69 & 47 & 116 \\
Total & & & \\
\hline
\end{tabular}

